\title{
ANHANG: DIALOG UND MÜNDLICHE SPRACHE
}

Literarische Dialoge sind zwar gelegentlich als mögliche Zeugen vergangener Gespräche untersucht worden, doch hat die Forschung die Schwierigkeit erkennen müssen, in literarischen Traditionen Hinweise zur vergangenen Mündlichkeit zu finden ${ }^{1}$. Im Laufe der Arbeit haben sich einige Beobachtungen zu diesem Thema ergeben, die hier zusammengefasst werden. In erster Linie fällt auf, dass nur sehr wenige Dialogautoren behaupten, mit ihrem Werk ein reales Gespräch wiederzugeben. Viel häufiger sind Aussagen, die sich auf die gewählte Form als rein schriftliche Darstellungsform eines Themas beziehen. In Kapitel 1 wurde eine Reihe von Zitaten aus den Prologen angeführt, in denen angekündigt wird, ein bestimmtes Thema werde per interrogationes et responsiones, per modum dialogi erörtert ${ }^{2}$.

Einige wenige Autoren von Streitgesprächen zwischen Christen und Juden behaupten, mit ihrem Werk eine wirkliche Diskussion wiederzugeben. Zum ersten Mal kommt diese Aussage bei Anselms Schüler Gilbert Crispin vor. Seine Darstellung der Umstände, wie es zu freundschaftlichen Diskussionen mit einem jüdischen Kaufmann kam, auf denen sein Dialogus basieren soll, ist vor dem bekannten historischen Hintergrund glaubwürdig ${ }^{3}$. Allerdings dürfte sein Text nur davon angeregt worden sein, nicht den genauen Verlauf einer konkreten Diskussion wiedergeben. Auch Odo von Cambrai sagt, einige der in seinem Dialogus dargelegten Überlegungen zur Menschwerdung Gottes habe er in einem Disput mit einem Juden namens Leo vorgebracht. Die Details, die er dazu gibt, sind mager, darunter ist aber eine Anmerkung, die Aufmerksamkeit erregt. Am Schluss des Dialogs, in dem die Figur Odo die Diskussion eindeutig dominiert, sagt der Verfasser, einige der anwesenden Christen hätten den Juden Leo unterstützt und ihn, Odo, dazu gezwungen, einige Punkte genauer zu diskutieren, als er vorhatte ${ }^{4}$. Wenn die Diskussion frei erfunden ist, sollte eine solche Anmerkung

\footnotetext{
${ }^{1}$ Siehe oben $12-13$.

2 Siehe oben $43-47$.

${ }^{3}$ Siehe oben $111-112$.

${ }^{4}$ Has, frater Acarde, Fudaeo reddidi rationes de adventu Christi, cogentibus me quaedam subtilius disputare quibusdam Catholicis qui intererant pro fudei parte (PL 160, 1112).
} 
wohl lediglich das Übergewicht der nur die christliche Seite interessierenden Argumente entschuldigen. Wenn der Dialog aber auf eine echte Diskussion zurückgeht, war es offensichtlich für den Verfasser zweitrangig, ihren tatsächlichen Verlauf wiederzugeben, denn in dem Werk kommen keine weiteren Figuren außer Odo und Leo vor. Petrus von Cornwall ist ein weiterer Verfasser, der eine reale Diskussion mit einem Juden als Ursprung seines Dialogs vorgibt und hinzufügt, der jüdische Disputant sei daraufhin zum Christentum übergetreten. Selbst wenn dies stimmt, dürfte auch für Petrus gelten, dass er solche Gespräche als Ausgangspunkt nimmt, nicht jedoch den tatsächlichen Verlauf getreu wiedergibt. In seinem Text (wie in den zwei vorherigen) finden sich vor allem lange, gelehrte Lehrvorträge des Christen und nur wenige Fragen und Einwände des Juden. Da es sich um spontane Gespräche gehandelt haben soll, nicht um reglementierte Disputationen wie diejenigen des Spätmittelalters, scheint diese einseitige Verteilung der Redeanteile kaum realistisch.

Unter den Streitgesprächen findet sich ein weiterer Text, der sich so wenig literarischen Konventionen fügt, dass er durchaus Spuren wirklicher Gespräche enthalten könnte: die Disputatio des Inghetto Contardo. Die Sprache hat einen eigenwilligen Duktus, der sich häufig der Volkssprache nähert, und erinnert mit ihrer assoziativen Argumentation, den Gedankensprüngen, den gegenseitigen Anreden und den Redundanzen an die mündliche Ausdrucksweise. Doch ist ein Caveat notwendig: Auch in dem Fall, dass die Gespräche wirklich stattfanden, formulierte Inghetto seinen Text einige Zeit später und aus dem Gedächtnis. Außerdem scheint es wahrscheinlich, dass er sich mit den mallorquinischen Juden in der Volkssprache (im italienischen Volgare und im Altkatalanischen, die mit Sicherheit gegenseitig verständlich waren) unterhielt. Es dürfte ergiebiger sein, im Text nicht nach Spuren der Mündlichkeit, sondern nach Interaktionsformen zu suchen. Inghetto schildert, wie die Personen sich grüßen oder meiden, ins Gespräch kommen, das Gespräch beenden oder abbrechen.

Das Verhältnis zwischen realer und literarischer Kommunikation, die in den Streitgesprächen vorzuherrschen scheint, besteht darin, dass reale Situationen die Anregung für die Dialogform geben, aber in ihr nicht wirklich abgebildet werden. Dies dürfte auch für die monastischen Lehrdialoge gelten und sogar für einige der selbstbetrachtenden Dialoge. Zum monastischen Leben gehörten mündliche Unterweisungssituationen - die Einweisung des Novizen durch den erfahrenen Mönch oder die monastische Predigt - sowie Beichtgespräche, die zur Kenntnis 
und Bewertung von sich selbst und von der eigenen Lebensführung anleiten sollten. In der Vita Hariolfi Ermenrichs von Ellwangen heißt der unterweisende Mönch Mahtolfus, wie ein realer Mönch, der Hariolf noch gekannt hatte. Mit dieser Benennung der Figur dürfte Ermenrich seine Informationsquelle angeben wollen (und dadurch ihre Zuverlässigkeit unterstreichen), ähnlich wie Mussato in seiner Evidentia, in der er Lovato Lovati auftreten lässt. Doch all diese Texte scheinen sich formell und inhaltlich in erster Linie an literarischen Traditionen zu orientieren; es gibt in Sprache, Stil und Inhalt nichts, was an ein mündliches Gespräch erinnert. Die Ausnahme ist ein Text, der sich ähnlich wie Inghettos Disputatio - in keine vorgegebene Form pressen lässt: die Visiones Richalmi abbatis. Auch hier sind zahlreiche sprachliche Kennzeichen der Umgangssprache zu entdecken, hinzu kommen auch indirekte Hinweise auf Umgangsformen bei Gesprächen.

Im Allgemeinen verwenden die Werke eine hieratische, formalistische Sprache. Die Ausnahmen sind - neben den gerade erwähnten Werken vornehmlich Texte, die den Einfluss der Theatersprache aufweisen: Eberhard von Ypern, der die Komödien als Vorbild nimmt, und zwei Kenner von Senecas Tragödien, Eugenius Vulgarius und Albertino Mussato. In den humanistischen Texten macht sich die Suche nach einer flüssigeren Ausdrucksweise bemerkbar, doch dürfte dies vor allem der Lektüre klassischer Modelle entstammen. Die Werke, die die Personensprache dramatisch gestalten, könnten auch reale Interaktionsformen abbilden wollen. Die Satansprozesse dürften zum Beispiel Umgangsformen vor Gericht wiedergeben. Bei Eberhard von Ypern finden sich Reflexionen und Beobachtungen über den korrekten sprachlichen Umgang, wie von Moos nachgewiesen hat ${ }^{5}$.

Man muss abschließend feststellen, dass die Dialogform vor allem eine Form der schriftlichen Kommunikation ist und als solche wahrgenommen und gehandhabt wurde. Nur in einigen Nischen finden sich Spuren der Mündlichkeit. Etwas ergiebiger dürften die Texte sein, in denen die Personensprache dramatisch gestaltet ist, vor allem wenn man nach Mustern der mündlichen Interaktion, und nicht nur nach konkreter mündlicher Sprache, sucht. Unter diesem Blickwinkel dürfte sich eine genauere Durchsicht lohnen.

5gl. von Moos, „Literatur- und bildungsgeschichtliche Aspekte“, 20-23; ders., „Le dialogue“, 365-368. 